\title{
20th Anniversary of the National Clinical Pharmacology Meeting at Giens
}

Under the proposal of the French Ministry of Health (Directorate of Medicines and Pharmacy) and the Ministry of Research (Delegation for Scientific Research and Technology), the National Clinical Pharmacology Meeting at Giens, the so-called 'Giens workshop', was created in 1984. In 2004, the 20th anniversary of this meeting was celebrated with the attendance of almost 200 medical experts from academia and the health care industry, and included large hospitals and the health care insurance system. During this meeting, two out of the three founding fathers of the Giens meeting honored the meeting with their presence, Professors Jacques Daugoumau and Pierre Simon. They remembered, in their words to the meeting attendees, Professor Jean-Paul Cano, the third founding member, who left us prematurely.

The goal of the National Clinical Pharmacology Meeting at Giens is to foster fruitful thinking and exchanges of option on pending issues in the field of clinical pharmacology, from a clinical as well as a methodological or regulatory stand point. These meetings are a privileged setting in which experts from several different fields can present and discuss their points of view without any pressure from their institutions or hierarchy. As a consequence, contacts are made between institutions, companies and competent authorities in a spirit of partnership and cooperation. In this setting, where any individual can express him- or herself on his or her own behalf, free from any 'Malthusian' restrictions that might be applied in his or her usual work environment, progresses can generally be made more rapidly and efficiently, especially in the case of sensitive issues, which classical 'institutional meetings' may fail to advance.

Thus, this meeting began, year after year, to be seen as a driving force for brainstorming and the development of new proposals, a force that could be unique in the French, and may be even in the European, clinical pharmacology and public health landscape.

In 2004, the 20th meeting took place with the sponsorship and partnership of the French Institute for Medical Research (INSERM), the French agency for health products (Afssaps), the Paris Public Hospital network (APHP), the National Public Health Insurance (CNAMTS), the representative institution of French drug companies (Leem-Recherche), the French Society of Pharmacology (SFP) and the Marseille Public Hospital Network (AP-HM)

In 2005, the Directorate for Public Health (DGS) at the French Ministry for health, family and social issues decided to join the sponsoring committee. In October, the Federation of French Hospitals (FHF) will join the committee as well.

These commitments come from major players in the fields of public health and research and development and are further evidences of the vitality, usefulness and attractiveness of the meeting.

The meeting is usually held over three days and is preceded by one or two preparatory meetings with subgroups. During the meeting at Giens, in each of their separate workshops, the subgroup attendees applied their knowledge and experience to answer a predetermined question submitted to them by the sponsoring committee and the scientific advisory councils. The questions had set criteria and concerned unresolved issues in public health research, ethics and the organisation of medical research in academia and industry.

The goal of the workshops is to speed up the resolution of issues in the area of evaluation and use of health products. Calls for action are proposed as conclusions. Two chairmen and one coordinator run the discussions.

The numbers of academic, institutional and private attendees are carefully assessed and balanced during the selection and invitation processes, as recommended by the sponsoring committee, the scientific advisory council and the foster council. The invited attendees are selected for their personal skills and/or knowledge and, therefore, their opinions do not necessarily reflect those of their institutions.

At the end of the meeting, the discussions and the proposals of all of the workshops are presented and challenged during a plenary session. A summary and the action lists are posted on the Giens workshops website (http://www.ateliersdegiens.org). The latter are published in this issue of Thérapie. We hope and wish, dear readers, that you will enjoy reading these reports and that those who hold a stake in public health will consider how to better progress towards our common goal of achieving better public health care.

Christian Libersa (General Secretary)

Daniel Vasmant (Chairman) 